\title{
Lateral Knee Pain after Outside-in Anatomic Double- Bundle Anterior Cruciate Ligament Reconstruction Using the TightRope RT
}

\author{
Kohei Kawaguchi, $\mathrm{MD}^{1}$, So Kuribayashi, $\mathrm{MD}^{1}$, Shuichi Nakayama, $\mathrm{MD}^{1}$, Keisuke Nakazato, $\mathrm{MD}^{1}$, \\ Toru Fukubayashi, $\mathrm{MD}, \mathrm{PhD}^{2}$, and Shuji Okinaga, $\mathrm{MD}^{1}$ \\ ${ }^{1}$ Department of Orthopaedic Surgery, Tokyo Teishin Hospital, Tokyo; ${ }^{2}$ Faculty of Sport Sciences, University of Waseda, Saitama, Japan
}

\begin{abstract}
The anterior cruciate ligament (ACL) TightRope RT (TR) was recently introduced as a novel cortical suspension device for ACL reconstruction. It has an adjustable graft loop that gives the surgeon some advantages during ACL reconstruction. We report three patients who required removal of the TR after an outside-in anatomical ACL reconstruction because of lateral knee pain. We assumed that the knee pain was associated with friction between the TR button of the posterolateral bundle and iliotibial band (ITB). Placing the TR button close to the lateral epicondyle and tissue interposition between the TR button and lateral femoral cortex may be potential risk factors for ITB irritation. Therefore, we recommend not placing the TR button close to the top of the lateral epicondyle and reducing the tissue interposition between the TR button and lateral femoral cortex as much as possible.
\end{abstract}

Keywords: Knee, Anterior cruciate ligament, Reconstruction, Outside-in technique, Suspensory fixation device

Double-bundle anterior cruciate ligament (ACL) reconstruction with the hamstring tendon has been widely performed ${ }^{1}$. Various techniques have been developed for fixing a graft to the femur, and several fixation devices are available for use during ACL reconstructions including cortical suspension devices, transfixation devices, and interference screws. Cortical suspension devices have been widely used because of their technical ease and rigidity ${ }^{2)}$. In contrast, femoral suspensory fixation devices may have encountered some problems, including irritation by the iliotibial band (ITB), tunnel enlargement, intra-articular migration, or intra-tunnel fixation ${ }^{3,4)}$.

An ACL TightRope RT (TR; Arthrex, Naples, FL, USA) was

Received April 16, 2015; Revised June 1, 2015;

Accepted June 21, 2015

Correspondence to: Kohei Kawaguchi, MD

Department of Orthopaedic Surgery, Tokyo Teishin Hospital, 2-14-23

Fujimi, Chiyoda-ku, Tokyo 102-8798, Japan

Tel: +81-3-5214-7111, Fax: +81-3-5214-7391

E-mail: kkohei0602@yahoo.co.jp

This is an Open Access article distributed under the terms of the Creative Commons Attribution Non-Commercial License (http://creativecommons.org/licenses/by-nc/4.0/) which permits unrestricted non-commercial use, distribution, and reproduction in any medium, provided the original work is properly cited. recently introduced as a novel cortical suspension device. Its adjustable graft loop affords the surgeon some freedom in terms of the length of the femoral socket, eliminates the requirement for bothersome intraoperative calculations for selecting loop length, ensures that the socket is completely filled with the graft, and provides the possibility of tightening the graft even after fixation $^{5,6)}$. To the best of our knowledge, there have been no previous reports of complications associated with TR in ACL reconstruction. In this report, we describe three cases of lateral knee pain after anatomical double-bundle ACL reconstruction using TR.

\section{Case Reports}

\section{Case 1}

A 29-year-old female was treated in our clinic in 2012 because of an ACL rupture. She had twisted and injured her knee during landing in karate. On preoperative physical examination, her Lachman test score was 3+ and a KneeLax examination (Monitored Rehab Systems BV, Haarlem, Netherlands), in which a 134 $\mathrm{N}$ force was applied, revealed a 2-mm slippage of the affected knee compared with the unaffected knee. The diagnosis of ACL tear was confirmed using magnetic resonance imaging (MRI). 
She underwent arthroscopic anatomical double-bundle reconstruction using hamstring tendons. The semitendinosus was harvested and divided into the anteromedial (AM) and the posterolateral (PL) bundles. We inserted the ACL Flipcutter (Arthrex) guide from the anterolateral portal to create femoral tunnels by outside-in technique. The Flipcutter was used as a retrograde drill ${ }^{5}$. The guide was set at $110^{\circ}$ for both the AM and PL tunnels. A stab incision was made at a point where the Flipcutter drill sleeve contacted the femoral lateral skin. Then, a $2.4-\mathrm{mm}$ guide wire was inserted to confirm that the position of the pin was appropriate, which was followed by alternate insertion of a $3.5-\mathrm{mm}$ guide wire and the Flipcutter. Retrograde drilling was performed using the Flipcutter to a length of $10 \mathrm{~mm}$ to create the socket. Two tibial tunnels for the AM and PL tunnels were created at $50^{\circ}$. After creating two femoral and two tibial tunnels, each graft was passed through each tunnel, and TR was used as the femoral fixation device.

Postoperative computed tomography (CT) revealed that the distance between the TR button and the exit of the tunnel on the lateral femoral cortex was $0 \mathrm{~mm}$ for the AM bundle and $10 \mathrm{~mm}$ for the PL bundle (Fig. 1). The knee was fixed in a brace for 1 week, after which the patient was instructed to perform a series of routine motion exercises. At 2 weeks after surgery, she felt slight lateral knee pain but she was able to perform her rehabilitation protocol with ITB stretching. However, at four months after surgery, she could only flex her knee to $90^{\circ}$ and extend to $-10^{\circ}$, and she was unable to perform the required rehabilitation exercises because of severe lateral pain. The lateral pain appeared when she flexed her knee more than $80^{\circ}$. A local anesthetic injection to the ITB relieved the pain slightly, but the lateral pain did not disappear and her rehabilitation exercise was heavily restricted. Therefore, we decided to remove the TR button of the PL bundle. At four months after the ACL surgery, we split the ITB and removed the TR button of the PL bundle. This button was located just beneath the ITB, but we were unable to assess the ITB in detail, because the skin incision was too small for close observation. In addition, we confirmed that the PL graft was fixed rigidly to the femoral tunnel although four months was a little early for graft-tunnel fixation. After the second surgery, the lateral pain immediately disappeared. Therefore, we considered that the pain was related to irritation between the TR button of the PL bundle and the ITB. A third arthroscopic evaluation was performed 14 months after the first ACL surgery, which showed that the reconstructed ACL had good synovial coverage and rigid tensioning. The patient was finally able to perform karate well.

\section{Case 2}

A 20-year-old male was treated in our clinic in 2012 because of an ACL rupture. He had twisted and injured his knee during landing while playing rugby. Preoperative physical examination revealed a Lachman test score of 3+ and a KneeLax examination, in which a $132 \mathrm{~N}$ force was applied, showed a 5-mm slippage of the affected knee compared with the unaffected knee. The diagnosis of ACL tear was confirmed by MRI. He underwent arthroscopic anatomic double-bundle reconstruction using hamstring tendons and the surgical procedure was the same as that
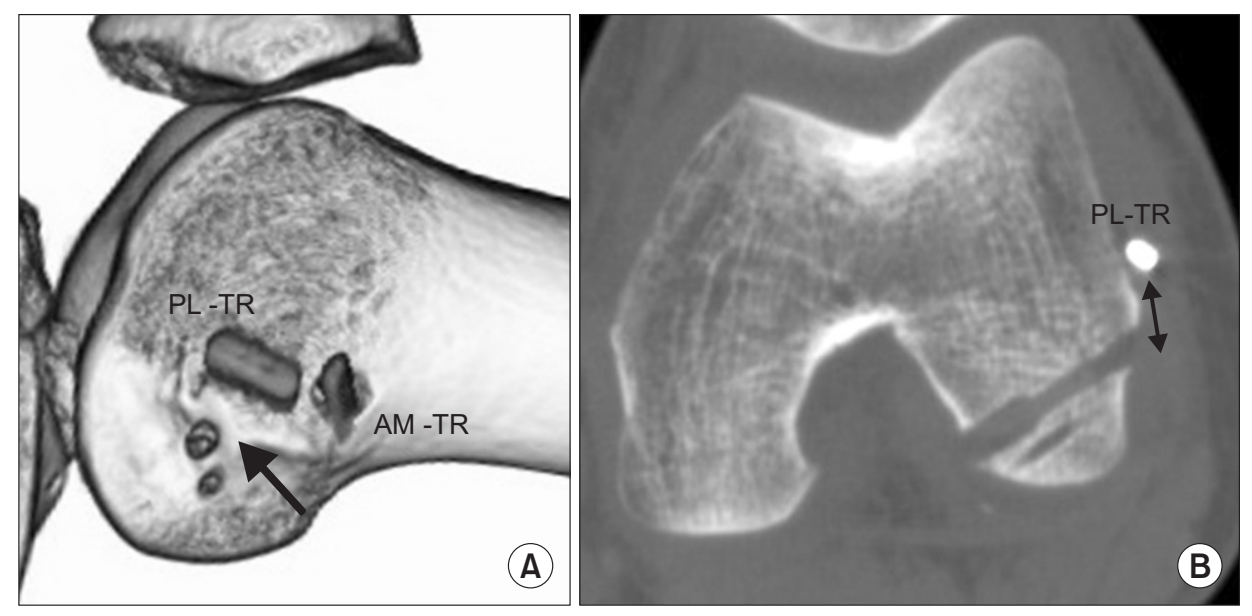

Fig. 1. Computed tomography images of case 1. (A) Three-dimensional reconstruction of the lateral femoral side. TightRope RT (TR) button of the posterolateral (PL) bundle is PL-TR and TR button of the anteromedial (AM) bundle is AM-TR. The black arrow indicates the lateral epicondyle of the femur. The TR button of the PL bundle is placed near the lateral epicondyle. (B) Transverse image at the level of the PL tunnel exit in the lateral femur showing that tissue interposition between the TR button and the femoral tunnel of the PL bundle. The black arrow indicates the distance between the TR button of the PL bundle and exit of the PL tunnel on the lateral femoral cortex. 
described in case 1.

Postoperative CT showed that the distance between the TR button and the exit of the tunnel on the lateral femoral cortex was 0 $\mathrm{mm}$ for the AM bundle and $1.6 \mathrm{~mm}$ for the PL bundle (Fig. 2). He undertook a rehabilitation exercise following routine procedures. At six months after the operation, the patient experienced moderate lateral knee pain on the TR button of the PL bundle and he was neither able to continue his rehabilitation routine nor return to playing rugby. The lateral pain appeared when he flexed his knee more than $120^{\circ}$ only in rehabilitation exercise. We decided to remove the TR button. A second arthroscopic evaluation of the graft indicated that the reconstructed ACL had good synovial coverage and rigid tensioning and the TR buttons of both AM and PL bundles were removed; the TR button of the PL bundle was just beneath the ITB. After the button's removal, the graft tensioning and incorporation were unchanged. After the second operation, his lateral knee pain immediately disappeared. Therefore, we considered that the pain was related to the irritation between the TR button of the PL bundle and the ITB as in case 1 . He was able to perform routine rehabilitation exercises and return to playing rugby 8 months after the first operation.

\section{Case 3}

A 19-year-old female was treated in our clinic in 2012 because of an ACL rupture. She had twisted and injured her knee in the guard position during a judo match. Preoperative physical examination revealed a Lachman test score of 3+ and a KneeLax examination, in which a $132 \mathrm{~N}$ force was applied, showed a 10mm slippage of the affected knee compared with the unaffected one. The diagnosis of ACL tear was confirmed using MRI. The patient underwent arthroscopic anatomic double-bundle reconstruction using hamstring tendons in a surgical procedure that was the same as that described in case 1.

Postoperative CT revealed that the distance between the TR button and the exit of the tunnel on the lateral femoral cortex was 0 $\mathrm{mm}$ for the AM bundle and $0.5 \mathrm{~mm}$ for the PL bundle (Fig. 3).
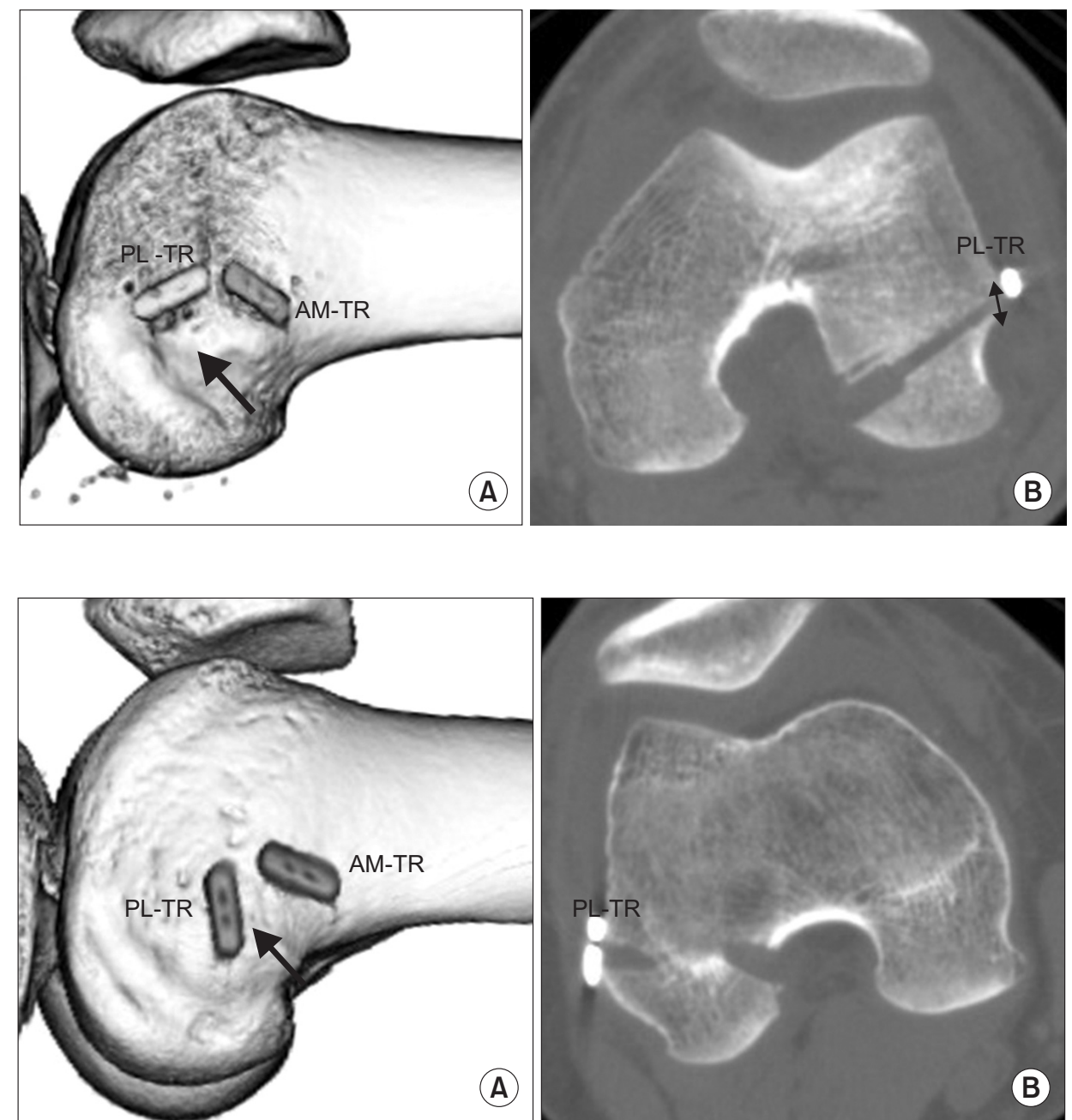

Fig. 2. Computed tomography images of case 2. (A) View of the lateral femur showing the TightRope (TR) button of the posterolateral (PL) bundle placed near the lateral epicondyle. The black arrow indicates the lateral epicondyle of the femur. (B) Transverse view showing slight tissue interposition between the TR button and femoral tunnel of the PL bundle. The black arrow shows the distance between TR button and exit of the PL tunnel on the lateral femoral cortex. AM: anteromedial.

Fig. 3. Computed tomography images of case 3. (A) View of the lateral femur showing the TightRope (TR) button of the poeterolateral (PL) bundle placed near the lateral epicondyle. The black arrow indicates the lateral epicondyle of the femur. (B) Transverse view showing no tissue interposition between the TR button of the PL bundle and PL femoral tunnel. AM: anteromedial. 
She undertook routine rehabilitation. At six months after the operation, she felt a slight lateral knee pain on the TR button of the PL bundle. However, she was able to carry out her routine rehabilitation schedule and return to playing judo. One year after the surgery, she could play judo and participate in competitions, but the lateral pain did not disappear and a slight swelling occurred on the lateral side of the knee. The lateral pain was not dependent on the knee flexion angle. She wanted to remove the TR buttons at the time of the second arthroscopy. TR buttons of both AM and PL bundles were removed; that of the PL bundle was just beneath the ITB. In addition, the adjustable loop in the TR of the PL bundle had partially worn out, and therefore was easily removed. A second arthroscopic evaluation of the graft indicated that the reconstructed ACL had good synovial coverage and rigid tensioning and the graft had been incorporated into the femoral bone tunnel. After removal of the TR button, graft tensioning and incorporation did not change and the lateral knee pain immediately disappeared. Therefore, we considered that the lateral pain was related to irritation between the TR button of the PL bundle and the ITB as in cases 1 and 2. The patient could finally return to performing judo.

This study had Institutional Review Board approval and informed consent was obtained from the patients.

\section{Discussion}

Some reports have described complications of ACL reconstruction using the conventional femoral suspensory fixation device, Endobutton CL (EB; Smith \& Nephew Endoscopy, Andover, MA, USA $)^{3,4)}$. However, to the best of our knowledge, there have been no previous reports on complications of ACL with the use of TR, a new femoral suspensory fixation device.

During the past three years, we have performed primary anatomical ACL reconstruction in 175 patients: outside-in anatomic double-bundle reconstructions using TR in 55 patients, insideout single-bundle reconstruction using TR in 50 patients, insideout double-bundle reconstruction using EB in 31 patients, outside-in single-bundle reconstruction using TR for augmentation in 21 patients, and outside-in double-bundle reconstruction using Retrobutton (Arthrex) in 18 patients. The three patients who required removal of the femoral fixation device underwent outside-in double-bundle reconstruction using TR. The overall removal rate after ACL reconstruction was $1.7 \%$; however, the rate was $5.4 \%$ in patients with outside-in double-bundle reconstruction. Therefore, we think this should not be overlooked as a low risk complication.
We believe that the lateral knee pain in the patients presented in this study was related to irritation between the TR of the PL bundle and the ITB. The pain was around the TR button of the PL bundle in all the patients, and the symptoms were similar to ITB syndrome. At the time of TR button removal, visual observations revealed that the ITB was very close to the TR button of the PL bundle. Taketomi et al. ${ }^{3)}$ reported two cases of ITB friction after double-bundle ACL reconstruction using EB and observed thickening of the ITB. They recommended that the button should not be placed on the lateral epicondyle. In the three cases described in the current report, TR buttons of the PL bundle had also been placed on the lateral epicondyle. Muhle et al. ${ }^{7)}$ reported that the ITB approached the lateral side of the femur nearest the level of the lateral epicondyle during a mild knee-flex. Therefore, the TR button of the PL bundle should not be placed on the lateral epicondyle, considering that the three cases required device removal.

However, we have seldom observed a removal of EB or Retrobutton, even when they were placed on the lateral epicondyle. We observed a high rate of TR button removal compared with the conventional femoral suspensory fixation devices, and this could be attributable to the button shape or the adjustable graft loop that may have tucked the soft tissue between the button and the lateral cortex.

As mentioned above, the lateral knee pain in the three cases was assumed to be caused by irritation between the TR button of the PL bundle and the ITB. Therefore, the distance from the ITB and the button or device is important. The distance between the ITB and button is defined by the location of the button and tissue interposition between the button and lateral femoral cortex. Mae et al. ${ }^{8)}$ reported that there was no relationship between tissue interposition and clinical symptoms in ACL reconstruction. However, the presence of tissue interposition between the TR button and femoral cortex shortens the distance between that button and the ITB, increasing the possibility of friction between them. In addition, the presence of interposition may influence the tucking of the adjustable loop. Two of our three cases had tissue interposition, which suggests that such interposition between the TR button and lateral femoral cortex should be avoided. Severe tissue interposition as in case 1 might cause severe and early complication. In contrast, slight interposition might cause delayed lateral knee pain as in case 2 and 3. Nag and Gupta ${ }^{6}$ reported on the surgical skill of flipping the TR button under arthroscopic visualization. Recently, we flipped the TR button using fluoroscopy and tried to remove the tissue under the button, if the fluoroscopy had shown tissue interposition. 
ACL TR is a novel cortical suspension device with advantageous adjustable graft loops ${ }^{5}$; however, the strength of the adjustable graft loop has not yet been established. Barrow et al. ${ }^{9)}$ were concerned regarding the potential disadvantages of the adjustable length design, such as loop lengthening after graft fixation. They showed that the mechanical strength of the cyclic loading of the TR was weaker than that of fixed-length cortical suspension devices, such as the EB. Consistent with this, Eguchi et al. ${ }^{10)}$ showed that the EB provided greater mechanical strength than the TR. In case 3 , we experienced partial rupture of the adjustable loop, although it was unclear when this rupture had occurred. We assumed that it had occurred after the incorporation of the graft with the bone tunnel for two reasons. First, the ACL graft tension was maintained during the second arthroscopy. Second, the lateral knee swelling occurred after the patient returned to judo one year after the surgery. The partial rupture gave the TR mobility, which led to swelling. During judo, the patient rubbed her knee against the floor during groundwork techniques, which may have led to the partial rupture of the adjustable loop.

In conclusion, we recommend not placing the TR button close to the top of the lateral epicondyle and reducing tissue interposition between the TR button and lateral femoral cortex as much as possible.

\section{Conflict of Interest}

No potential conflict of interest relevant to this article was reported.

\section{References}

1. Taketomi S, Inui H, Nakamura K, Hirota J, Sanada T, Masuda $\mathrm{H}$, Takeda $\mathrm{H}$, Tanaka S, Nakagawa T. Clinical outcome of anatomic double-bundle ACL reconstruction and 3D CT model-based validation of femoral socket aperture position. Knee Surg Sports Traumatol Arthrosc. 2014;22:2194-201.

2. Kamelger FS, Onder U, Schmoelz W, Tecklenburg K, Arora $\mathrm{R}$, Fink C. Suspensory fixation of grafts in anterior cruciate ligament reconstruction: a biomechanical comparison of 3 implants. Arthroscopy. 2009;25:767-76.

3. Taketomi S, Inui H, Hirota J, Nakamura K, Sanada T, Masuda H, Tanaka S, Nakagawa T. Iliotibial band irritation caused by the EndoButton after anatomic double-bundle anterior cruciate ligament reconstruction: report of two cases. Knee. 2013;20:291-4.

4. Fauno P, Kaalund S. Tunnel widening after hamstring anterior cruciate ligament reconstruction is influenced by the type of graft fixation used: a prospective randomized study. Arthroscopy. 2005;21:1337-41.

5. Lubowitz JH, Ahmad CS, Anderson K. All-inside anterior cruciate ligament graft-link technique: second-generation, no-incision anterior cruciate ligament reconstruction. Arthroscopy. 2011;27:717-27.

6. Nag HL, Gupta H. Seating of TightRope RT button under direct arthroscopic visualization in anterior cruciate ligament reconstruction to prevent potential complications. Arthrosc Tech. 2012;1:e83-5.

7. Muhle C, Ahn JM, Yeh L, Bergman GA, Boutin RD, Schweitzer M, Jacobson JA, Haghighi P, Trudell DJ, Resnick D. Iliotibial band friction syndrome: MR imaging findings in 16 patients and MR arthrographic study of six cadaveric knees. Radiology. 1999;212:103-10.

8. Mae T, Kuroda S, Matsumoto N, Yoneda M, Nakata K, Yoshikawa H, Shino K. Migration of EndoButton after anatomic double-bundle anterior cruciate ligament reconstruction. Arthroscopy. 2011;27:1528-35.

9. Barrow AE, Pilia M, Guda T, Kadrmas WR, Burns TC. Femoral suspension devices for anterior cruciate ligament reconstruction: do adjustable loops lengthen? Am J Sports Med. 2014;42:343-9.

10. Eguchi A, Ochi M, Adachi N, Deie M, Nakamae A, Usman MA. Mechanical properties of suspensory fixation devices for anterior cruciate ligament reconstruction: comparison of the fixed-length loop device versus the adjustable-length loop device. Knee. 2014;21:743-8. 